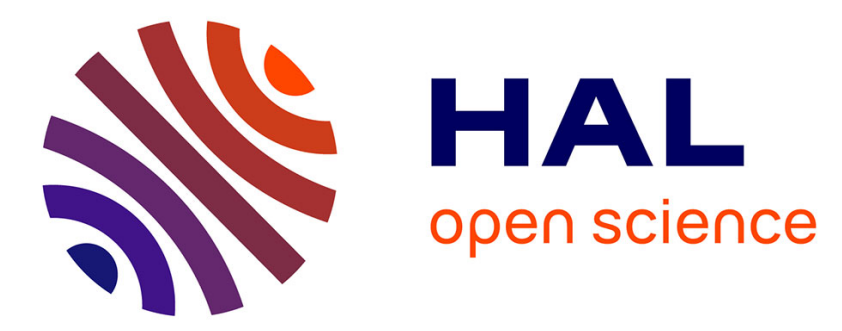

\title{
Superposition with Equivalence Reasoning andDelayed Clause Normal Form Transformation
}

\author{
Harald Ganzinger, Jürgen Stuber
}

\section{To cite this version:}

Harald Ganzinger, Jürgen Stuber. Superposition with Equivalence Reasoning andDelayed Clause Normal Form Transformation. [Research Report] RR-4835, INRIA. 2003, pp.23. inria-00071750

\section{HAL Id: inria-00071750 \\ https://hal.inria.fr/inria-00071750}

Submitted on 23 May 2006

HAL is a multi-disciplinary open access archive for the deposit and dissemination of scientific research documents, whether they are published or not. The documents may come from teaching and research institutions in France or abroad, or from public or private research centers.
L'archive ouverte pluridisciplinaire HAL, est destinée au dépôt et à la diffusion de documents scientifiques de niveau recherche, publiés ou non, émanant des établissements d'enseignement et de recherche français ou étrangers, des laboratoires publics ou privés. 
INSTITUT NATIONAL DE RECHERCHE EN INFORMATIQUE ET EN AUTOMATIQUE

\title{
Superposition with Equivalence Reasoning and Delayed Clause Normal Form Transformation
}

\author{
Harald Ganzinger — Jürgen Stuber
}

$\mathbf{N}^{\circ} 4835$

Mai 2003

THÈME 2 



\title{
Superposition with Equivalence Reasoning and Delayed Clause Normal Form Transformation
}

\author{
Harald Ganzinger* , Jürgen Stuber ${ }^{\dagger}$ \\ Thème 2 - Génie logiciel \\ et calcul symbolique \\ Projets PROTHEO \\ Rapport de recherche $\mathrm{n}^{\circ} 4835$ - Mai 2003 - 23 pages
}

\begin{abstract}
This report describes a superposition calculus where quantifiers are eliminated lazily. Superposition and simplification inferences may employ equivalences that have arbitrary formulas at their smaller side. A closely related calculus is implemented in the Saturate system and has shown useful on many examples, in particular in set theory. The report presents a completeness proof and reports on practical experience obtained with the Saturate system.
\end{abstract}

Key-words: first-order logic, automated deduction, equivalence, equality, resolution, superposition, clause normal form transformation, set theory

\footnotetext{
* MPI für Informatik, 66123 Saarbrücken, Germany. Email: hg@mpi-sb.mpg.de

$\dagger$ LORIA École des Mines de Nancy, 615 Rue du Jardin Botanique, 54600 Villers-lès-Nancy, France. Email: stuber@loria.fr
} 


\section{Superposition avec raisonnement sur des equivalences et transformation paresseuse en forme clausale}

Résumé : Ce rapport décrit un calcul de superposition qui élimine les quantificateurs paresseusement. Les inférences de superposition et simplification peuvent utiliser des équivalences logiques avec des formules arbitraires du coté inférieur. Un calcul étroitement lié est implanté dans le système Saturate. Son utilité est montrée grâce à de nombreux exemples, en particulier en théorie des ensembles. Le rapport présente une preuve de complétude et expose des expériences pratiques menées avec le système Saturate.

Mots-clés : logique du premier ordre, déduction automatique, équivalence, égalité, résolution, superposition, transformation clause normale, théorie des ensembles 


\section{Introduction}

"Translating a conjecture into clause normal form before handing it over to the theorem prover is like shooting oneself into the foot before starting on a long hike." With these or similar words G. Huet alluded to the fact that clause normal form transformation may obscure the logical structure to an extent making subsequent proof search very difficult, if not impossible [10]. This paper describes an approach to automated first-order theorem proving based on superposition where a compromise is sought between preserving formula structure, in particular quantifiers, and performing logical reasoning, in particular formula simplification, efficiently on clausal data structures. More specifically, we will describe and prove refutationally complete a clausal inference system, where literals in clauses can be equations between terms or equivalences between arbitrary first-order formulas. Term equations give rise to the usual superposition inferences, and so do equivalences when the larger side in the formula ordering is an atomic formula. The smaller side of an equivalence can be any formula. If the larger side of an equivalence literal is a nonatomic formula and if that literal is selected for inference, the formula is simplified by an analytic expansion rule. This system allows one to reason with equivalences as they might arise from definitions or lemmas in a natural way, and in particular use orientable equivalences as simplification rules, even if they are guarded by side conditions in a clausal context.

A calculus closely related to what we describe below was implemented in the Saturate system [9] a few years ago and has proved to be useful in an encouraging number of cases. We have carried out practical experiments on various formalizations of fragments of set theory and from a proof of the Church-Rosser property of the lambda calculus. Experience shows that the proof search becomes more goal-oriented and generates much fewer clauses until a proof is found. Although Saturate is comparatively slow, this allows it to be competitive with Vampire [15] and E-Setheo [16] on the set theory examples that were used in the first-order division of the last CASC competition [6].

The work in this paper can be viewed as an extension of the extended narrowing calculus $[8,18]$ whereby not only initial equivalences can be used for simplification, but also those that have side conditions (contextual simplification) and those generated dynamically during the saturation process. Our completeness proof reuses some of the constructions of the latter paper. [7] also presents a method for handling definitions more efficiently, attempting to recover equivalences from clauses and treating them with specific selection strategies for inferences. Admitting inferences on nonclausal formulas, the calculus described below is also related to the various calculi of nonclausal resolution and superposition $[11,12,2,4]$. The main difference here is that we also admit quantifiers, and that the local nature of classical superposition inferences is better preserved, facilitating the development of efficient implementations of our calculus in a theorem prover. In [1] the author argues, by discussing some examples, that a tight integration of sequent calculus, tableaux, and resolution should be beneficial to proof search, but does not propose any concrete calculus in this regard.

The work started with the implementation efforts in the Saturate system, and now revisited in a theoretical setting, should also be viewed as a first step towards closing the large gap in logical notation used in interactive theorem provers for higher-order logic at one 
side, and present-day automated provers à la E-Setheo, SPASS, or Vampire at the other side of the spectrum. That gap needs to be closed for any successful integration of automated provers into interactive ones. To close that gap even further, first-order provers should also understand some restricted higher-order notation. Steps into this direction might be facilitated with our present results.

\section{A Motivating Example}

Consider the definition of extensionality on sets by

$$
\text { eq }(A, B) \Leftrightarrow \text { ! X. (contains }(A, X) \Leftrightarrow=\operatorname{contains}(B, X))
$$

where ! is the universal quantifier. A typical clause normal form of this equivalence (and saturated with respect to superposition by Saturate) would be this (with $\mathbf{s} \$ 3$ denoting a Skolem function, and "*" denoting a clause in which the first negative literal is selected):

* 4(2) : eq $(B, C)$, contains (B,D) $\rightarrow$ contains (C,D)

* 5(2) : eq (B,C), contains (C,D) $\rightarrow$ contains (B,D)

9(4): contains $(B, s \$ 3(C, B))$, contains $(C, s \$ 3(C, B))$, eq $(C, B)$

* 10(4) : contains $(B, s \$ 3(C, B))$, contains $(C, s \$ 3(C, B)) \rightarrow$ eq $(C, B)$

19(8) : eq $(B, B)$

Having these clauses in the context of other axioms for set theory can create a quite large search space with many redundancies. Treating equivalence equationally by rewriting and superposition is much better. This is the proof of the transitivity of eq (line 2) that Saturate finds (about 15 times faster than the purely clausal proof, and by purely deterministic steps) with an inference system such as the one proposed in this paper:

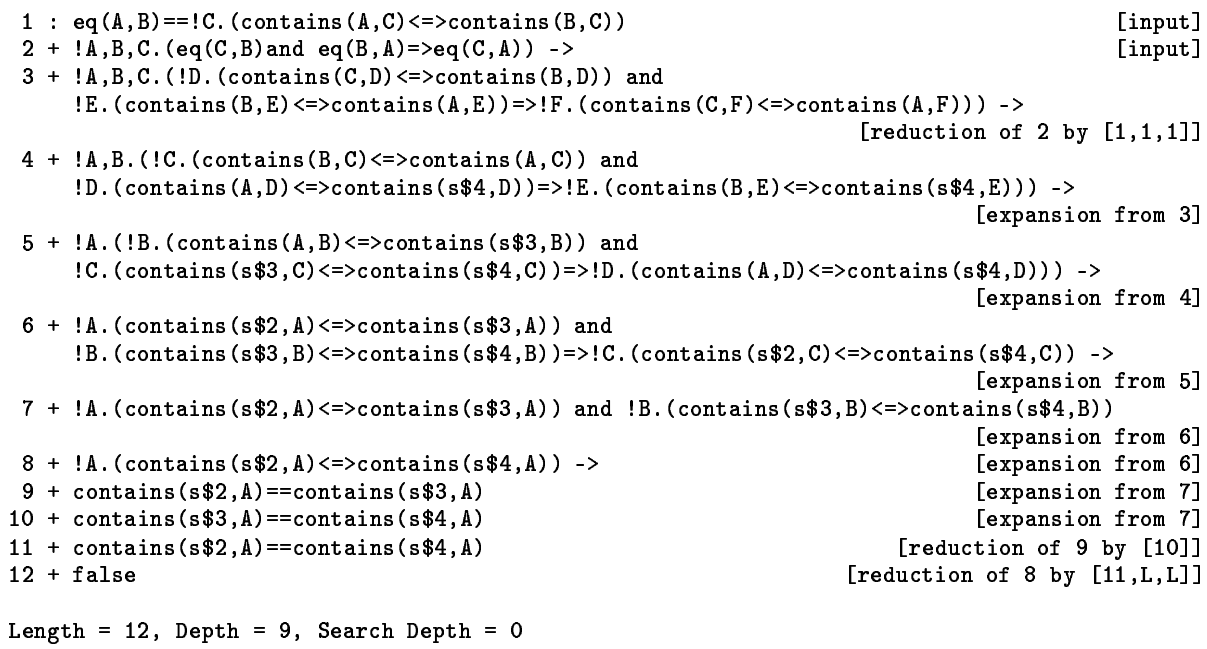


Steps $3-10$ expand the goal by rewriting with the definition of eq in 1 , by applying analytic rules in the sequent calculus, and by skolemization. After this the proof finishes by rewriting using the equivalences $1,9,10$, and 11 , with "==" denoting structural equivalence (or equality on the propositional level). This proof is found without any search at all as each inference is simplifying. Note that some equivalences are in the input explicitly, and others come to the surface by proof search. This short proof was made possible by rewriting with equivalences such as clause 1 in which the larger side is a standard atom, but where the smaller side in the ordering can be any formula, possibly including quantifiers.

The fact that with the clausal form of the extensionality axiom the search space can be very large is demonstrated by the nontermination of provers including Saturate and SPASS on trivially consistent clause sets such as the one resulting by adding the definition of union

contains (union $(A, B), X) \Leftrightarrow \operatorname{contains}(A, X)$ or $\operatorname{contains}(B, X)$

to the extensionality clauses. With the inference system proposed here, Saturate will orient both equivalences, the one for eq and the one for union, from left to right, and terminate immediately as no critical pair exists.

\section{Language}

We assume as given a first-order language consisting of a set of predicate symbols $\mathcal{P}$ and a set of function symbols $\mathcal{F}$, and an arity function $\alpha: \mathcal{P} \cup \mathcal{F} \rightarrow \mathbb{N}$. $\mathcal{F}$ has to contain sufficiently many function symbols for skolemization. We also assume a countably infinite set of variables $\mathcal{X}$. We will implicitly use deBruijn notation for bound variables to obtain a canonical representation for formulas, however, we will usually write formulas in the standard way. In deBruijn notation bound variables are represented by natural numbers $\geq 1$, the deBruijn indices, where $n$ refers to the $n$-th quantifier above $n$. For example, $\forall x \exists y p(x, y)$ is written as $\forall \exists p(2,1)$. We assume that deBruijn indices are modified appropriately whenever necessary, for example when quantifiers are removed. Terms will be built in the usual way from function symbols (which include constants as function symbols of arity 0), free variables from $\mathcal{X}$ and bound variables from $\mathbb{N} \geq 1$. A nonequational atom is an atom of the form $p\left(t_{1}, \ldots, t_{n}\right)$ where $p$ is a predicate symbol of arity $n$ and $t_{1}, \ldots, t_{n}$ are terms. An equation is written $s \approx t$. An atom is either a nonequational atom or an equation. A firstorder formula is built from nonequational atoms and equations, using the logical operators $\perp$ (false), $\top$ (true), $\vee, \wedge, \rightarrow, \approx$ (equivalence), $\forall$ and $\exists$. We use $\neg F$ as an abbreviation for $F \rightarrow \perp$. We say a formula is trivial if it is either $\top$ or $\perp$. Note that we use the same symbol for equality and for logical equivalence which, for soundness, implicitly requires a two-sorted approach. We let $\approx$ be symmetric, i.e., we do not distinguish $l \approx r$ and $r \approx l$, both for equations and for equivalences. We write $F={ }_{s} G$ to indicate that $F$ and $G$ are equal up to symmetry of $\approx$.

A (generalized) literal is an equation or an equivalence. Note that an arbitrary logical formula $F$ can be expressed as the literal $F \approx T$. Finally, a (generalized) clause is a multiset of literals. We write $\square$ for the empty clause, and we will omit the multiset braces around 
singletons. For example, we will write $L \cup C$ for the clause consisting of the literal $L$ and the literals in $C$.

We call the signature consisting of $\mathcal{P}$ and $\mathcal{F}$ the base signature, while the full signature containing the base signature and all the logical operators will be the extended signature. A clause or formula is closed if it contains no free variables, and ground if it contains no variables (i.e. not even deBruijn indices). A ground instance of a clause, formula or term is an instance obtained by substituting terms over the base signature for free variables. So in general ground instances are not ground, only closed. Note that ground instantiation does not introduce new bound variables. We write $\left\{t_{1} / x_{1}, \ldots, t_{n} / x_{n}\right\}$ for the substitution that replaces $x_{i}$ by $t_{i}$ for $i \in\{1, \ldots, n\}$.

The Herbrand universe $\mathrm{HU}$ is the set of ground terms over the base signature, and the Herbrand base HB is the set of all nonequational ground atoms over the base signature plus all equations between terms in the Herbrand universe. A (nonequational Herbrand) interpretation $I$ is a subset of the Herbrand base. A ground atom $A$ is true in $I$ if and only if $A \in I$. A quantified formula $\forall x F$ is true if $F\{t / x\}$ is true in $I$ for all ground terms $t$. Analogously $\exists x F$ is true if there exists a ground instantiation of $x$ that is true in $I$. Other logical operators are interpreted as usual, which extends the interpretation to all closed clauses. Clauses with free variables will be considered true in $I$ if all its closed instances are true in $I$, i.e. free variables are considered to be universally quantified.

\section{Ordering}

In order to do the model construction and obtain refutational completeness we need a wellordering on closed clauses with the following properties:

1. The ordering has to be a total simplification ordering on closed terms and formulas that is compatible with the symmetry of $\approx$.

We can achieve this by an RPO over a total precedence that has multiset status for $\approx$ and lexicographic status for the other symbols.

2. The ordering must decrease when a quantified variable is instantiated by an arbitrary ground term, so deBruijn indices need to be larger than all ground terms over $\mathcal{F}$.

In the precedence we let deBruijn indices be greater than function symbols.

3. $T$ is the least and $\perp$ the next-to-least element.

We put $\perp \succ T$ at the bottom of the precedence.

4. To make the factoring inference decreasing we need that for $s$ a term or a nonequational atom, $s \succ t_{1}$ and $s \succ t_{2}$ imply $s \approx t_{1} \succ t_{1} \approx t_{2}$.

In the precedence we let $\approx$ be smaller than all function and predicate symbols.

The following precedence is suitable for our purposes:

$$
\cdots \succ 2 \succ 1 \succ 0 \succ f \succ \approx \succ \rightarrow \succ \forall \succ \exists \succ \vee \succ \wedge \succ \perp \succ \top
$$

Here numbers stands for the bound variables in deBruijn notation, and $f$ for an arbitrary well-ordering on all function symbols. Predicate symbols may be placed arbitrarily above $\approx$ 
in the precedence. This particular choice of a precedence has the additional advantage that it orients the CNF transformation rules of Stuber [18], which can be useful for simplification and refinements of the calculus. We denote the RPO obtained from such a precedence by $\succ$.

We extend $\succ$ from terms and first-order formulas to literals by considering a literal $l \approx r$ as the multiset $\{l, r\}$ and comparing them by using the multiset extension of $\succ$. Clauses are multisets of their literals and are again naturally compared by the multiset extension of the literal ordering. We say a literal $L$ is maximal in a clause $L \cup C$ if $L \succeq L^{\prime}$ for all literals $L^{\prime}$ in $C$.

We will uniformly write $\succ$ for the orderings on terms, on formulas, on literals, and on clauses. When we compare a literal and a clause we consider the literal as a unit clause.

\section{The Inference System}

We begin by describing some properties that all our inferences will have. Inferences have a main premise $C$, a conclusion $D$, and zero or more side premises $C_{1}, \ldots, C_{n}$. Such an inference is written

$$
\frac{C \quad C_{1} \quad \ldots \quad C_{n}}{D},
$$

where we will always write the main premise first. The inference operates on the main literal in the main premise, which we will write as the first literal of the clause. A side premise $C_{i}$ always has the form $l_{i} \approx r_{i} \cup C_{i}^{\prime}$, and we assume that variables are renamed to be distinct from those of the other premises. Inferences on closed clauses, which we call closed inferences, will have the additional implicit side conditions that the main premise is greater than the side premises and the conclusion, that the main literal is maximal in the main premise, and that $\left(l_{i} \approx r_{i}\right) \succ C_{i}^{\prime}$ and $l_{i} \succ r_{i}$ for each side premise $C_{i}$. For the actual nonground inferences we approximate the ordering, for example $\succ$ by $\npreceq$. In inferences where a substitution (i.e. an mgu) is applied the comparison is made on the substituted clauses.

First we present the Elimination inference rules that expand clauses to clause normal form. To write them more concisely we adapt the unified notation of Smullyan to our literal syntax:

\begin{tabular}{c|cc}
$\alpha$ & $\alpha_{1}$ & $\alpha_{2}$ \\
\hline$F \wedge G \approx \top$ & $F \approx \top$ & $G \approx \top$ \\
$F \vee G \approx \perp$ & $F \approx \perp$ & $G \approx \perp$ \\
$F \rightarrow G \approx \perp$ & $F \approx \top$ & $G \approx \perp$
\end{tabular}

\begin{tabular}{c|cc}
$\beta$ & $\beta_{1}$ & $\beta_{2}$ \\
\hline$F \wedge G \approx \perp$ & $F \approx \perp$ & $G \approx \perp$ \\
$F \vee G \approx \top$ & $F \approx \top$ & $G \approx \top$ \\
$F \rightarrow G \approx \top$ & $F \approx \perp$ & $G \approx \top$
\end{tabular}

\begin{tabular}{c|c}
$\gamma$ & $\gamma(t)$ \\
\hline$\forall x F \approx \top$ & $F\{t / x\} \approx \top$ \\
$\exists x F \approx \perp$ & $F\{t / x\} \approx \perp$
\end{tabular}

\begin{tabular}{c|c}
$\delta$ & $\delta(t)$ \\
\hline$\forall x F \approx \perp$ & $F\{t / x\} \approx \perp$ \\
$\exists x F \approx \top$ & $F\{t / x\} \approx \top$
\end{tabular}

$\perp$ Elimination $\quad \frac{\perp \approx \top \cup C}{C}$ 
$\alpha$ Elimination $\quad \frac{\alpha \cup C}{\alpha_{i} \cup C} \quad$ for $i \in\{1,2\}$.

$\beta$ Elimination $\quad \frac{\beta \cup C}{\beta_{1} \cup \beta_{2} \cup C}$

$\gamma$ Elimination $\quad \frac{\gamma \cup C}{\gamma(z) \cup C}$

where $z$ is a fresh variable.

$\delta$ Elimination $\quad \frac{\delta \cup C}{\delta\left(f\left(x_{1}, \ldots, x_{n}\right)\right) \cup C}$

where $f$ is a fresh skolem function and $x_{1}, \ldots, x_{n}$ are the free variables in $\delta$.

$\approx \top$ Elimination $\quad \frac{(l \approx r) \approx \top \cup C}{l \approx r \cup C}$

Positive Equivalence Elimination $1 \quad \frac{l \approx r \cup C}{l \approx \perp \cup r \approx \top \cup C}$

where $l$ and $r$ are nontrivial formulas, $l \not \preceq r$, and $l$ is not a nonequational atom.

Positive Equivalence Elimination $2 \quad \frac{l \approx r \cup C}{l \approx \top \cup r \approx \perp \cup C}$

where $l$ and $r$ are nontrivial formulas, $l \npreceq r$, and $l$ is not a nonequational atom.

Negative Equivalence Elimination $1 \quad \frac{(l \approx r) \approx \perp \cup C}{l \approx \top \cup r \approx \top \cup C}$

where $l$ and $r$ are formulas.

Negative Equivalence Elimination $2 \quad \frac{(l \approx r) \approx \perp \cup C}{l \approx \perp \cup r \approx \perp \cup C}$

where $l$ and $r$ are formulas.

The second part of the calculus is a two-sorted superposition calculus, which is very similar to standard superposition.

Reflexivity Resolution $\quad \frac{(s \approx t) \approx \perp \cup C}{C \sigma}$

where (i) $s$ and $t$ are terms, and (ii) $\sigma$ is a most general unifier of $s$ and $t$.

Negative Superposition $\quad \frac{\left(s\left[l^{\prime}\right] \approx t\right) \approx \perp \cup C \quad l \approx r \cup D}{((s[r] \approx t) \approx \perp \cup C \cup D) \sigma}$

where (i) $s\left[l^{\prime}\right]$ and $t$ are terms, (ii) $l^{\prime}$ is not a variable, (iii) $\sigma$ is a most general unifier of $l$ and $l^{\prime}$, and (iv) $s\left[l^{\prime}\right] \sigma \npreceq t \sigma$. 
Positive Superposition $\frac{s\left[l^{\prime}\right] \approx t \cup C \quad l \approx r \cup D}{(s[r] \approx t \cup C \cup D) \sigma}$

where (i) $l^{\prime}$ is not a variable, (ii) $\sigma$ is a most general unifier of $l$ and $l^{\prime}$, (iii) $s\left[l^{\prime}\right] \sigma \npreceq t \sigma$, and (iv) $\left(s\left[l^{\prime}\right] \approx t\right) \sigma \npreceq C \sigma$.

$\approx$ Factoring

$$
\frac{l \approx r \cup l^{\prime} \approx r^{\prime} \cup C}{\left(\left(r \approx r^{\prime}\right) \approx \perp \cup l^{\prime} \approx r^{\prime} \cup C\right) \sigma}
$$

where (i) $\sigma$ is a most general unifier of $l$ and $l^{\prime}$, and (ii) $l \sigma \npreceq r \sigma \nprec r^{\prime} \sigma$.

We let ES denote this set of inferences.

If we restrict the input to a set of clauses containing only literals of the forms $s \approx t$ and $(s \approx t) \approx \perp$ where $s$ and $t$ are terms, none of the Elimination inferences is applicable, and we recover a syntactic variant of the standard superposition calculus [14], with exactly the same ordering restrictions. However, if we consider clause sets containing only literals of the form $A \approx \top$ or $A \approx \perp$, we do not get the standard ordered resolution calculus. Instead we need factoring on both positive and negative literals and obtain a resolution inference derived from Positive Superposition that restricts both resolved literals to be strictly maximal. Nevertheless, a calculus extended by selection (see Section 7) can force negative literals to be treated by Negative Superposition, which leads to a syntactical variant of standard ordered resolution with selection.

\section{Refutational Completeness}

We prove the refutational completeness of ES in the presence of strong redundancy criteria using the reduction-of-counterexamples framework of Bachmair and Ganzinger [4]. We assume that the reader is familiar with this method.

We will construct a Herbrand model by well-ordered recursion over $\succ$ on closed clauses. We construct a rewrite system $R$ that contains rules of the form $l \Rightarrow r$ where either $l$ and $r$ are ground terms, or where $l$ is a nonequational ground atom and $r$ is a closed formula. We will write $s \Rightarrow_{R} t$ or just $s \Rightarrow t$ when $s$ rewrites to $t \stackrel{\leftrightarrows}{\Rightarrow}$ for the transitive closure of $\Rightarrow$, $\stackrel{*}{\Rightarrow}$ for the reflexive-transitive closure of $\Rightarrow$, and $s \Downarrow t$ if $s \stackrel{*}{\Rightarrow} u \stackrel{*}{\Leftarrow} t$. We will construct $R$ in such a way that all left-hand sides of rules are irreducible by other rules, which implies confluence. To ensure termination we will require $l \succ r$ for all rules.

Suppose $R$ is such a rewrite system. We define the rewrite closure of $R$, written $R^{*}$, as the smallest set of closed first-order formulas that satisfies the following conditions:

1. If $F \stackrel{\leftrightarrows}{\Rightarrow} G$ and $G \in R^{*}$ then $F \in R^{*}$.

2. $\top \in R^{*}$.

3. $s \approx s \in R^{*}$ for all ground terms $s$.

4. If $F \in R^{*}$ and $G \in R^{*}$ then $F \wedge G \in R^{*}$.

5. If $F \in R^{*}$ or $G \in R^{*}$ then $F \vee G \in R^{*}$.

6. If $F \notin R^{*}$ or $G \in R^{*}$ then $F \rightarrow G \in R^{*}$. 
7. If $F \in R^{*}$ iff $G \in R^{*}$ then $F \approx G \in R^{*}$.

8. If $F\{t / x\} \in R^{*}$ for all ground terms $t$ then $\forall x F \in R^{*}$.

9. If $F\{t / x\} \in R^{*}$ for some ground term $t$ then $\exists x F \in R^{*}$.

$R^{*}$ can be understood as a minimal (first-order, equality) Hintikka set containing $R$. This definition is by induction over $\succ$. In particular in case (6), the only one involving negation, the formulas in the condition are smaller than the formula in the conclusion, so $R^{*}$ is welldefined. By restricting $R^{*}$ to the Herbrand base it becomes a Herbrand interpretation, and we obviously have $F \in R^{*}$ if and only if $\mathrm{HB} \cap R^{*}=F$. In the following we will also write $R^{*}$ for this Herbrand interpretation.

Lemma 1 The set of term equations in $R^{*}$ is a congruence.

Proof: It follows from the use of a confluent rewrite system and from the logical properties of equivalence.

Lemma 2 Let $l \Rightarrow r$ be a rule in $R$. Then $R^{*}=l \approx r$.

Proof: We can rewrite $l \approx r$ to $r \approx r$, which is in $R^{*}$ by reflexivity.

We assume as given a set $N$ of clauses and build an interpretation, intended to be a model of $N$, from the ground instances of $N$. For each closed clause $C$ we will define a set $R_{C}$ of rewrite rules derived from clauses smaller than $C$, the Hintikka set $I_{C}=R_{C}^{*}$ derived from $R_{C}$, and a set $\Delta_{C}$ that contains either a rewrite rule derived from $C$ or is empty. The definition of $\Delta_{C}$ depends on $R_{C}$ and $I_{C}$; it is well-defined, since $R_{C}$ and $I_{C}$ only depend on $\Delta_{D}$ for $D$ smaller than $C$. Formally we let

$$
\begin{aligned}
& \Delta_{C}= \begin{cases}\{l \Rightarrow r\} \quad \text { if (i) } I_{C} \not \neq C^{\prime}, \\
& \text { (ii) } C=l \approx r \cup C^{\prime} \text { is a ground instance of a clause in } N, \\
& \text { (iii) } l \text { and } r \text { are terms, or } l \text { is a nonequational atom, } \\
& \text { (iv) }(l \approx r) \succ C^{\prime}, \\
& \text { (v) } l \succ r, \\
& \text { (vi) } l \text { is irreducible by } R_{C}, \text { and } \\
\text { (vii) }(R \cup\{l \Rightarrow r\})^{*} \not \neq C^{\prime} \text {; or }\end{cases} \\
& R_{C}=\bigcup_{D \prec C} \Delta_{D} \\
& I_{C}=R_{C}^{*}
\end{aligned}
$$

To obtain an interpretation for all ground instances of $N$ we let

$$
R_{N}=\bigcup_{C} \Delta_{C} \quad \text { and } \quad I_{N}=R_{N}^{*} .
$$

We say that a closed clause $C$ produces $l \Rightarrow r$ in $I_{N}$ if $l \Rightarrow r$ is in $\Delta_{C}$ for the set of clauses $N$. In that case we say that $C$ is productive in $I_{N}$.

INRIA 
Lemma 3 If $C=l \approx r \cup C^{\prime}$ produces $l \Rightarrow r$ then $C^{\prime}$ is false in $I_{N}$.

Proof: Suppose $C^{\prime}$ is true in $I_{N}$. Then some closed clause $D \succeq C$ produces a rule $l^{\prime} \Rightarrow r^{\prime}$ that reduces $C^{\prime}$. By condition (vii) this rule cannot be the one produced by $C$ itself, so $D \succ C$. Because $l^{\prime}$ is irreducible w.r.t. $l \Rightarrow r$ we have $l^{\prime} \succ l$. But no term or formula in $C^{\prime}$ is greater than $l$, so $C^{\prime}$ cannot be reduced by $l^{\prime} \Rightarrow r^{\prime}$ and we have a contradiction.

We now come to the proof of the reduction property of ES, which is the main part in the proof of refutational completeness. We call a closed clause $C$ a counterexample for $I_{N}$ if it is a ground instance of a clause in $N$ and false in $I_{N}$. Since closed clauses are well-ordered by $\succ$, there exists a least counterexample whenever $I_{N} \not \models N$. Let $C$ be a counterexample for $I_{N}$ and let $\mathcal{I}$ be a closed inference with main premise $C$, side premises $C_{1}, \ldots, C_{n}$ and conclusion $D$. We say that $\mathcal{I}$ reduces the counterexample $C$ (with respect to $I_{N}$ ) if $I_{N} \models \neg D$, $C \succ D$ and the side premises are productive in $I_{N}$. An inference system Calc has the reduction property for counterexamples (with respect to $I$ ) if there is a ground instance of an inference in Calc that reduces $C$ for $I_{N}$ for any set $N$ of ground clauses such that $I_{N}$ has the least counterexample $C \neq \square$.

Lemma 4 ES has the reduction property for counterexamples.

Proof: Let $N$ be a set of clauses, let $C$ be the least counterexample in $I_{N}$ and suppose $C \neq \square$. Then $C=L \cup C^{\prime}$ with $L=l \approx r$ maximal in $C$. Since $C$ is false in $I_{N}$, we have $l \neq{ }_{s} r$, and we may assume $l \succ r$ without loss of generality. $C$ is not productive, as $l \Rightarrow r \in R_{N}$ would imply $I_{N} \mid=l \approx r$. We do case analysis on the possible causes, i.e. the condition on $\Delta_{C} \neq \emptyset$ that is violated:

(iii) Suppose $l$ is a formula, but not a nonequational atom, i.e. $l$ has a logical operator at its root. We do a case analysis over the form of $l$ and $r$.

(iii.1) $l$ cannot be $T$, as $T$ is minimal in $\succ$ and $r$ must be smaller.

(iii.2) Suppose $l=\perp$. Then $r=\top$, and the $\perp$ Elimination inference

$$
\frac{\perp \approx \top \cup C^{\prime}}{C^{\prime}}
$$

reduces $C$.

(iii.3) Suppose $L$ is an $\alpha$-formula. Since $L$ is false in $I_{N}$, so $\alpha_{1}$ or $\alpha_{2}$ is false in $I_{N}$, and one of the $\alpha$ Elimination inferences

$$
\frac{\alpha \cup C^{\prime}}{\alpha_{1} \cup C^{\prime}} \quad \text { or } \quad \frac{\alpha \cup C^{\prime}}{\alpha_{2} \cup C^{\prime}}
$$

reduces $C$.

(iii.4) Suppose $L$ is a $\beta$-formula. Since $L$ is false in $I_{N}$, both $\beta_{1}$ and $\beta_{2}$ are false and the $\beta$ Elimination inference

$$
\frac{\beta \cup C^{\prime}}{\beta_{1} \cup \beta_{2} \cup C^{\prime}}
$$


reduces $C$.

(iii.5) Suppose $L$ is a $\gamma$-formula. Since $L$ is false in $I_{N}$, there exists a ground term $t$ such that $\gamma(t)$ is false in $I_{N}$. Hence the closed inference

$$
\frac{\gamma \cup C^{\prime}}{\gamma(t) \cup C^{\prime}}
$$

reduces $C$. The conclusion is smaller than $C$, since bound variables are larger in the ordering than any ground term over the base signature. If the variable doesn't occur in the formula the removal of the quantifier still makes the conclusion slightly smaller. This inference is a ground instance of $\gamma$ Elimination, where we choose to instantiate the fresh variable by $t$.

(iii.6) Suppose $L$ is a $\delta$-formula. $L$ being false in $I_{N}$ implies that for all ground terms $t$ over the base signature the formula $\delta(t)$ is false in $I_{N}$. Let $\sigma$ be the substitution used to derive $C$ from a clause in $N$, and let $x_{1}, \ldots, x_{n}$ be the free variables in its literal corresponding to $L$. Then the ground instance

$$
\frac{\delta \cup C^{\prime}}{\delta\left(f\left(x_{1} \sigma, \ldots, x_{n} \sigma\right)\right) \cup C^{\prime}}
$$

of $\delta$ Elimination reduces $C$ by the same argument as in (iii.5). Note that $f$ is in the base signature and that freshness is not needed for the completeness proof.

(iii.7) Suppose $L=(s \approx t) \approx \top$. Then the $\approx \top$ Elimination inference

$$
\frac{(s \approx t) \approx \top \cup C^{\prime}}{s \approx t \cup C^{\prime}}
$$

reduces $C$.

(iii.8) Suppose $L=(s \approx t) \approx \perp$.

(iii.8.1) Suppose $s$ and $t$ are terms. Then $I_{N}=s \approx t$.

(iii.8.1.1) If $s=t$ then Reflexivity Resolution reduces $C$.

(iii.8.1.2) Otherwise without loss of generality $s \succ t$ and $s \Downarrow_{R_{N}} t$, so $s$ is reducible by $R_{N}$. We have $C=\hat{C} \sigma$ for some clause $\hat{C}$ in $N$ and a ground substitution $\sigma$, where $\hat{C}=(\hat{s} \approx \hat{t}) \approx \perp \cup \hat{C}^{\prime}$ with, among others, $\hat{s} \sigma=s$. If the reduction step were in a variable position in $\hat{s}$, then we could reduce $\sigma$ to $\tau$ and obtain a smaller ground instance $\hat{C} \tau$ of $N$ that would also be a counterexample of $I_{N}$, a contradiction to the minimality of $C$. So $\sigma$ must be irreducible, and the reduction is not in a variable position.

$s$ is reduced by some rule $l^{\prime} \Rightarrow r^{\prime}$ in $R_{N}$, which has been produced by some clause $D=l^{\prime} \approx r^{\prime} \cup D^{\prime}$. Since $l^{\prime}$ is a subterm of $s$ we have $s \succeq l$, which implies $l \succ l^{\prime}$ and in turn $C \succ D$, so $s$ is reducible already by $R_{C}$. We have the ground instance of Negative Superposition

$$
\frac{\left(s\left[l^{\prime}\right] \approx t\right) \approx \perp \cup C^{\prime} \quad l^{\prime} \approx r^{\prime} \cup D^{\prime}}{\left(s\left[r^{\prime}\right] \approx t\right) \approx \perp \cup C^{\prime} \cup D^{\prime}}
$$

which reduces $C$. The conclusion is smaller than $C$ due to monotonicity, and the main literal preserves its truth value since $l^{\prime} \approx r^{\prime}$ is valid in $I_{N}$ and since $\approx$ is interpreted by a congruence in $I_{N}$.

INRIA 
(iii.8.2) Otherwise $s$ and $t$ are formulas. Since $L$ is false in $I_{N}$ the equivalence $s \approx t$ is true, so either both $s$ and $t$ are true or both are false. Thus one of the Negative Equivalence Elimination inferences

$$
\frac{(s \approx t) \approx \perp \cup C^{\prime}}{s \approx \top \cup t \approx \top \cup C^{\prime}} \text { and } \frac{(s \approx t) \approx \perp \cup C^{\prime}}{s \approx \perp \cup t \approx \perp \cup C^{\prime}}
$$

has a conclusion that is false in $I_{N}$. The conclusions are also smaller, as the new literals can be embedded in $(s \approx t) \approx \perp$, so $C$ is reduced.

(iii.9) Otherwise both $l$ and $r$ are formulas distinct from $T$ and $\perp$, and $l$ is not a nonequational atom. Since $L$ is false in $I_{N}, s$ and $t$ have distinct truth values in $I_{N}$, and the Positive Equivalence Elimination inference

$$
\frac{s \approx t \cup C^{\prime}}{s \approx \perp \cup t \approx \top \cup C^{\prime}} \quad \text { or } \quad \frac{s \approx t \cup C^{\prime}}{s \approx \top \cup t \approx \perp \cup C^{\prime}}
$$

reduces $C$. In particular, as $s$ and $t$ are nontrivial the new literals become smaller than $s \approx t$.

(iv) From now on we may assume that (iii) holds, so $l$ is either a term or a nonequational atom. Suppose that $L$ is not strictly maximal in $C$, so that there is another literal $L^{\prime}$ in $C$ with $L={ }_{s} L^{\prime}$. Let $L^{\prime}=l^{\prime} \approx r^{\prime}$ with $l={ }_{s} l^{\prime}$ and $r={ }_{s} r^{\prime}$. The inference

$$
\frac{l \approx r \cup l^{\prime} \approx r^{\prime} \cup C^{\prime \prime}}{\left(r \approx r^{\prime}\right) \approx \perp \cup l^{\prime} \approx r^{\prime} \cup C^{\prime \prime}}
$$

is a ground instance of Equality Factoring. By property (4) of the ordering the literal $\left(r \approx r^{\prime}\right) \approx \perp$ is smaller than $l \approx r$, as $l$ has a function or predicate symbol at the root and $l \succ r, r^{\prime}$. By reflexivity of $\approx$ this literal is also false in $I_{N}$, so this inference reduces $C$.

(vi) From now on we may assume that (iv) holds, i.e. $L \succ C^{\prime}$. Suppose $L$ is reducible by $R_{C}$. Then $l=l\left[l^{\prime}\right]$ and $l^{\prime} \Rightarrow r^{\prime} \in R_{C}$ is produced by some clause $D=l^{\prime} \approx r^{\prime} \cup D^{\prime}$ with $l^{\prime} \succ r^{\prime}$ and $\left(l^{\prime} \approx r^{\prime}\right) \succ D^{\prime}$. By the same argument as in (iii.8.1.2) the ground substitution that leads to this instance is irreducible by $R_{N}$. We have the ground instance of Positive Superposition

$$
\frac{l\left[l^{\prime}\right] \approx r \cup C^{\prime} \quad l^{\prime} \approx r^{\prime} \cup D^{\prime}}{l\left[r^{\prime}\right] \approx r \cup C^{\prime} \cup D^{\prime}}
$$

which reduces $C$ by the same argument as in (iii.8.1.2).

(vii) Otherwise $\left(R_{C} \cup\{l \Rightarrow r\}\right)^{*} \models C^{\prime}$. Then $C^{\prime}=L^{\prime} \cup C^{\prime \prime}$ and $\left(R_{C} \cup\{l \Rightarrow r\}\right)^{*} \models L^{\prime}$, where $L \succeq L^{\prime} . L^{\prime}$ must contain $l$ as a subterm to be affected by the rule $l \Rightarrow r$, so $L^{\prime}=l^{\prime} \approx r^{\prime}$ with $l={ }_{s} l^{\prime} \succ r \succeq r^{\prime}$. For $L^{\prime}$ to become true in $\left(R_{C} \cup\{l \Rightarrow r\}\right)^{*}$ we must have $r \approx r^{\prime}$ true in $\left(R_{C} \cup\{l \Rightarrow r\}\right)^{*}$. This implies $I_{N}=r \approx r^{\prime}$, because no clause greater or equal than $C$ can produce a rule that affects $r$ or $r^{\prime}$. The inference

$$
\frac{l \approx r \cup l^{\prime} \approx r^{\prime} \cup C^{\prime \prime}}{\left(r \approx r^{\prime}\right) \approx \perp \cup l^{\prime} \approx r^{\prime} \cup C^{\prime \prime}}
$$

is a ground instance of Equality Factoring whose conclusion is smaller than $C$ by the same argument as in (iv), so it reduces $C$. 
We call a closed clause $C$ redundant in $N$ if there exist ground instances $C_{1}, \ldots, C_{k}$ of clauses in $N$ such that $C \succ C_{i}$ for $i=1, \ldots, k$ and $C_{1}, \ldots, C_{k}=C$. A (nonclosed) clause is redundant if all its ground instances are redundant.

For the reduction of a least counterexample by an inference redundant clauses are irrelevant. The least counterexample cannot be redundant, as being implied by smaller true clauses would contradict it being false. Any productive clause $D$ used as a side premise cannot be redundant either, as it would also be implied by smaller true clauses, which contradicts the condition for productivity of being false in $I_{D}$. Hence redundant clauses can be ignored in inferences.

We may simplify a clause $C$ to some clauses $D_{1}, \ldots, D_{n}$ in $N$ if these clauses follow logically from $N \cup\{C\}$ and if $C$ is redundant in $N \cup\left\{D_{1}, \ldots, D_{n}\right\}$. A closed inference

$$
\begin{array}{cccc}
C & C_{1} \quad \ldots \quad C_{n} \\
\hline D
\end{array}
$$

is redundant in $N$ if there exist ground instances $D_{1}, \ldots, D_{k}$ of clauses in $N$ such that $C \succ D_{i}$ for $i=1, \ldots, k$ and $D_{1}, \ldots, D_{k}, C_{1}, \ldots, C_{n} \models D$. Again, a (nonclosed) inference is redundant if all its ground instances are redundant. The following well-known lemma allows to delete redundant clauses without losing redundancy.

Lemma 5 Let $N$ be a set of clauses and $M$ the set of redundant clauses in $N$. If a clause $C$ is redundant in $N$ then it is redundant in $N \backslash M$.

Proof: Suppose a clause $\hat{C}$ is redundant in $N$ but not in $N \backslash M$. Then there exists a ground instance $C$ of $\hat{C}$ that is redundant in $N$ but not in $N \backslash M$. We choose $\hat{C}$ and $C$ such that $C$ is the least such ground instance. Since $C$ is redundant in $N$, there exist ground instances $D_{1}, \ldots, D_{n}$ of $N$ that imply $C$. Let $D_{1}, \ldots, D_{n}$ be the least set of such ground instances with respect to the multiset extension of $\succ$. Now by assumption some $D_{i}$ must be a ground instance of $M$ but not of $N \backslash M$. Since $D_{i}$ is smaller than $C$, it is redundant in $N \backslash M$ by induction hypothesis. That is, $D_{i}$ is in turn implied by smaller ground instances $D_{1}^{\prime}, \ldots, D_{m}^{\prime}$ of $N \backslash M$. We may replace $D_{i}$ by $D_{1}^{\prime}, \ldots, D_{m}^{\prime}$ to obtain a smaller multiset, in contradiction to the minimality of $D_{1}, \ldots, D_{n}$. Hence $D_{1}, \ldots, D_{n}$ must be ground instances of $N \backslash M$, and $C$ and $\hat{C}$ are redundant in $N \backslash M$.

A set $N$ of clauses is saturated with respect to ES if all inferences in ES with premises in $N$ are redundant. The reduction property implies refutational completeness for ES:

Theorem 6 Let $N$ be a set of clauses that is saturated with respect to ES. If $N$ is not satisfiable then $\square \in N$.

Proof: Suppose $N$ is saturated and not satisfiable, but $\square \notin N$. Then ES contains an inference with a ground instance

$$
\begin{array}{cccc}
C & C_{1} \quad \ldots \quad C_{n} \\
\hline D
\end{array}
$$


that reduces the least counterexample $C$ to some smaller closed clause $D$ that is false in $I_{N}$. By saturation this inference is redundant, so there exist ground instances $D_{1}, \ldots, D_{k}$ of clauses in $N$ such that $C \succ D_{i}$ for $i=1, \ldots, k$ and $D_{1}, \ldots, D_{k}, C_{1}, \ldots, C_{n} \models D$. Since $D$ is false in $I_{N}$ one of the closed clauses $D_{1}, \ldots, D_{k}, C_{1}, \ldots, C_{n}$, which are all smaller than $C$, must be false in $I_{N}$, in contradiction to the minimality of $C$.

For the $\delta$ Elimination inferences the conclusion is not a logical consequence of the premise, as Skolemization only preserves satisfiability. However, the conclusion implies the premise. Premise and conclusion become logically equivalent in the presence of the implication in the other direction:

$$
\forall x_{1}, \ldots, x_{n} .(\exists x . F) \rightarrow F\left\{f\left(x_{1}, \ldots, x_{n}\right) / x\right\}
$$

We call (1) the skolem axiom for the skolem function symbol $f$ with respect to $\exists x . F$. We call a set of skolem axioms $S$ fresh with respect to a set of propositions $N$ if no skolem function symbol of $S$ occurs in $N$, and there is only one skolem axiom for every skolem function symbol in $S$. A fresh set of skolem axioms is always obtained when fresh function symbols are used for skolemization.

Proposition 7 Let $N$ be a set of propositions and $S$ a set of skolem axioms that is fresh with respect to $N$, and let $I$ be a model of $N$. Then there exists a model $I^{\prime}$ of $N \cup S$.

Proof: Sketch: define the interpretation of skolem functions in $I^{\prime}$ so that they provide witnesses for the true instances of their corresponding existential formula. Since $S$ is fresh this is possible without changing the truth value of $N$.

For a suitable set of skolem axioms this proposition isolates the argument that skolemization preserves satisfiability, and allows us to use the simpler notion of logical equivalence elsewhere.

A theorem proving derivation with respect to a set $S$ of skolem axioms is a sequence of sets of clauses $N_{0} \vdash N_{1} \vdash \ldots$ such that for all steps $N_{i} \vdash N_{i+1}, i \geq 0$, either (1) $N_{i+1}=N_{i} \cup M$ for some set $M$ of clauses such that $N_{i} \cup S \models M$, or (2) $N_{i+1}=N_{i} \backslash M$ for some set $M$ of clauses such that each clause $C$ in $M$ is redundant in $N_{i}$. In case (1) we call the step a deduction step and in case (2) a deletion step. For such a derivation the set of persistent clauses $N_{\infty}$ is defined as $N_{\infty}=\bigcup_{i \geq 0} \bigcap_{j>i} N_{j}$. We will now show that certain properties are preserved when going from $N_{0}$ to the limit $N_{\infty}$ or vice-versa.

Lemma 8 Let $N_{0} \vdash N_{1} \vdash$. be a theorem proving derivation and let $I$ be some interpretation. If all ground instances of $N_{\infty}$ are true in I then all ground instances of $\bigcup_{i} N_{i}$ are true in $I$.

Proof: Consider some ground instance $C$ of some clause $\hat{C}$ in $\bigcup_{i} N_{i}$. If $\hat{C}$ is in $N_{\infty}$ then $C$ is true by assumption. Otherwise $\hat{C}$ has been removed by some deletion step $N_{i} \vdash N_{i+1}$, hence it is redundant in $N_{i}$ and thus in $\bigcup_{i} N_{i}$. Let $M$ be the set of all redundant clause in $\bigcup_{i} N_{i}$, then by the above argument $\left(\bigcup_{i} N_{i}\right) \backslash M \subseteq N_{\infty}$. Hence $C$ is redundant in $N_{\infty}$ by Lemma 5 , and there exist ground instances of $N_{\infty}$ that imply $C$ and are true in $I$. We conclude that $C$ is true in $I$. 
This implies in particular that all ground instances of $N_{0}$ are true in $I$, and that $N_{0}$ is satisfiable if $N_{\infty}$ is satisfiable.

Lemma 9 Let $N_{0} \vdash N_{1} \vdash$. . be a theorem proving derivation. with respect to a fresh set $S$ of skolem axioms. If $N_{0}$ is satisfiable then $N_{\infty}$ is satisfiable.

Proof: Suppose we are given a model $I_{0}$ of $N_{0}$. Then by Lemma 7 there exists a model $I$ of $N_{0} \cup S$. Furthermore, $N_{\infty} \subseteq \bigcup_{i} N_{i}$ contains only logical consequences of $N_{0} \cup S$, so we may conclude $I \models N_{\infty}$.

Corollary 10 Let $N_{0} \vdash N_{1} \vdash$. be a theorem proving derivation with respect to a fresh set $S$ of skolem axioms. Then $N_{0}$ is satisfiable if and only if $N_{\infty}$ is satisfiable.

A theorem proving derivation is called fair (with respect to ES) if all inferences in ES from clauses in $N_{\infty}$ are redundant in $N_{i}$ for some $i \geq 0$. Since the conclusions of ground inferences are always smaller than the main premise, and since they imply themselves, an inference can be made redundant by a deduction step that adds its conclusion. Thus a fair derivation can always be obtained by considering inferences in a fair way, i.e. not delaying an inference ad infinitum, and adding their conclusion if they are not already known to be redundant by some suitable sufficient criterion.

Lemma 11 Let $N_{0} \vdash N_{1} \vdash \ldots$ be a fair theorem proving derivation. Then $N_{\infty}$ is saturated up to redundancy.

Proof: We have to show that all inferences from clauses in $N_{\infty}$ are redundant in $N_{\infty}$. From the definition of fair we know that all inferences from clauses in $N_{\infty}$ are redundant in $\bigcup_{i} N_{i}$, and by Lemma 5 in $N_{\infty}$.

Theorem 12 Let $N_{0} \vdash N_{1} \vdash$.. be a fair theorem proving derivation. If $N_{0}$ is not satisfiable then $\square \in N_{\infty}$.

\section{$7 \quad$ Refinements}

As is the case with other superposition calculi we can apply various refinements.

Certain of our inferences, in particular all of the Elimination ones, are simplifications. Instead of using them exactly in the way the inference system suggests we may use other simplifications. For example, we may simplify $(A \approx F) \approx \perp$ to $A \approx \neg F$, as the two are logically equivalent and the second is smaller in $\succ$. On the other hand we may choose to expand some equivalences that are orientable, using Positive Equivalence Elimination as a simplification rule. We may use any equivalence that holds in the model and is orientable w.r.t. the ordering for rewriting inside a formula, for example the CNF transformation rules of Stuber [18], or equivalences in $N$. More generally we may even use clauses for contextual reduction of formulas. 


\begin{tabular}{|l|r|r|l|l||l|r|r|c|c|}
\hline Problem & \multicolumn{2}{|c|}{ Saturate } & V & ES & Problem & \multicolumn{2}{|c|}{ Saturate } & V & ES \\
& \#clauses & time/s & & & & & \#clauses & time/s & \\
\hline SET014+4 & 84 & 1.8 & - & - & SET630+3 & 3049 & 116.8 & + & + \\
SET017+1 & - & - & + & + & SET640+3 & 293 & 6.0 & + & + \\
SET067+1 & 138 & 5.8 & + & + & SET646+3 & - & - & + & + \\
SET076+1 & 161 & 4.4 & + & - & SET647+3 & 3360 & 236.0 & + & + \\
SET086+1 & 285 & 9.6 & + & + & SET648+3 & 3345 & 251.1 & + & + \\
SET096+1 & 214 & 8.1 & + & + & SET649+3 & - & - & + & + \\
SET108+1 & - & - & + & + & SET651+3 & - & - & + & + \\
SET143+3 & 107 & 2.0 & + & + & SET657+3 & 456 & 16.0 & + & + \\
SET143+4 & 71 & 1.1 & + & + & SET669+3 & - & - & + & + \\
SET171+3 & 152 & 2.9 & - & - & SET671+3 & - & - & + & - \\
SET580+3 & 95 & 1.7 & + & + & SET673+3 & - & - & + & - \\
SET601+3 & 207 & 5.3 & - & - & SET680+3 & 784 & 46.2 & + & + \\
SET606+3 & 93 & 1.3 & + & + & SET683+3 & 143 & 4.6 & + & - \\
SET607+3 & 77 & 1.1 & + & + & SET684+3 & - & - & + & - \\
SET609+3 & 136 & 2.1 & - & - & SET686+3 & - & - & + & + \\
SET611+3 & 325 & 11.0 & + & + & SET716+4 & 193 & 8.8 & - & - \\
SET612+3 & 145 & 9.9 & - & - & SET724+4 & 380 & 18.1 & - & - \\
SET614+3 & 126 & 9.5 & - & - & SET747+4 & - & - & - & - \\
SET615+3 & 203 & 11.1 & - & - & SET764+4 & 241 & 6.2 & + & + \\
SET624+3 & 172 & 10.2 & + & + & SET770+4 & 174 & 5.0 & - & - \\
\hline solved & 18 & & 13 & 12 & Solved & 11 & & 16 & 12 \\
\hline
\end{tabular}

Figure 1: Experimental results on the SET problems from the CASC-18 FOF competition (V: Vampire 5.0, ES: E-Setheo csp02)

Another modification is to add selection to the calculus. A formula $F$ is in a negative position in a clause $C$ if $F$ being false in $I$ implies that $C$ is true in $I$. For example, the equation in the Negative Superposition inference is in a negative position. We may select some equation in any negative position in a clause, and then apply suitably generalized Reflexivity Resolution and Negative Superposition inferences at this position, instead of any other inference with this clause. We may also select a nonequational atom and use equivalences to do superposition in a similar way. This refinement is complete, since any minimal counterexample with a selected atom can be reduced in a similar way as by Reflexivity Resolution and Negative Superposition in the proof above. We may further generalize this to the selection of more than one position, which leads to Hyperparamodulation and Hyperresolution inferences.

Theorem proving with built-in theories over terms $[3,17]$ should pose no new fundamental problems, as should adding the basic restriction $[5,13]$.

\section{Experiments}

A calculus that differs from the one described before only with respect to minor technicalities related to clause representation had been implemented previously in the Saturate system. The expansion inference rules are implemented in a structure preserving manner by introducing new names for the subformulas as suggested in [19]. Skolem functions are associated with each quantified subformula at parse time so that if that subformula becomes produced in different contexts the same function is used. One can choose between eager 
and lazy expansion of logical symbols other than equivalences. In the presence of structural expansion, eager expansion does not create duplication, and contextual rewriting, as it is implemented in Saturate, can discharge contexts better if they do not contain any general formulas.

In Figure 1 we summarize Saturate's performance on the 40 problems from the TPTP category SET that were chosen for the CASC-18 competition in the first-order division [6]. We compare Saturate's results with the performance of Vampire 5.0 and E-Setheo csp02 at that competition ("-" means no proof found at the competition with a time limit of 600 seconds; "+" means proof found). Saturate was run with a clause limit of 10000 clauses and a time limit of 300 seconds. All results were obtained in auto mode. In particular the ordering for orienting equivalences was computed without user interaction. An iterative deepening strategy for proof search was employed.

Saturate is an experimental system written in Prolog and is comparatively slow. Therefore the runtimes (in seconds on a $2 \mathrm{GHz}$ notebook) are not too interesting. What we want to emphasize is the relatively small number of clauses generated on those problems where Saturate finds the proof. Vampire typically generates up to several orders of magnitude more clauses than Saturate. As an extreme case, for proving SET143+3 Vampire generates almost 8 million clauses.

One should also say that most of these problems are trivial, so we really do want to find the proof without much search, and the iterative deepening (proof depth) strategy for inference selection turned out to be quite useful here. We believe the reason why Vampire and E-Setheo do not perform well on some of these examples is due to their inability to suitably treat equivalences occurring in extensionality axioms for set equality and the subset relation. Another problem with some of the examples is that they consist of a relatively large axiomatization where only few axioms are needed in each case for the proof of the respective goal. Saturate also has problems with redundant axiomatizations and small changes can have a big effect on proof finding.

\section{References}

[1] Arnon Avron. Gentzen-type systems, resolution and tableaux. Journal of Automated Reasoning, 10(2):265-281, 1993.

[2] Leo Bachmair and Harald Ganzinger. Non-clausal resolution and superposition with selection and redundancy criteria. In Logic Programming and Automated Reasoning, LNCS 624, pages 273-284. Springer, 1992.

[3] Leo Bachmair and Harald Ganzinger. Associative-commutative superposition. In Proc. 4th Int. Workshop on Conditional and Typed Rewriting, LNCS 968, pages 1-14, Jerusalem, 1994. Springer.

[4] Leo Bachmair and Harald Ganzinger. Resolution theorem proving. In Alan Robinson and Andrei Voronkov, editors, Handbook of Automated Reasoning, volume 1, chapter 2, pages 19100. North Holland, 2001. 
[5] Leo Bachmair, Harald Ganzinger, Christopher Lynch, and Wayne Snyder. Basic paramodulation. Information and Computation, 121(2):172-192, 1995.

[6] The CADE-18 ATP System Competition (CASC-18), 2002. http://www.cs.miami.edu/ $\sim \operatorname{tptp} / \mathrm{CASC} / 18 /$.

[7] Anatoli Degtyarev and Andrei Voronkov. Stratified resolution. In Proc. 17th Int. Conf. on Automated Deduction (CADE-17), LNAI 1831, pages 365-384, Pittsburgh, PA, USA, 2000. Springer.

[8] Gilles Dowek, Thérèse Hardin, and Claude Kirchner. Theorem proving modulo. Rapport de Recherche 3400, INRIA, 1998.

[9] Harald Ganzinger, Robert Nieuwenhuis, and Pilar Nivela. The Saturate system. System available at http://www.mpi-sb.mpg.de/SATURATE/, 1994.

[10] Gérard Huet. The zipper talk. Invited Lecture at FLoC'96, 1996.

[11] Z. Manna and R. Waldinger. A deductive approach to program synthesis. ACM Transactions on Programming Languages and Systems, 2(1), 1980.

[12] Neil V. Murray. Completely non-clausal theorem proving. Artificial Intelligence, 18(1):67-85, 1982.

[13] Robert Nieuwenhuis and Albert Rubio. Theorem proving with ordering and equality constrained clauses. Journal of Symbolic Computation, 19:321-351, 1995.

[14] Robert Nieuwenhuis and Albert Rubio. Paramodulation-based theorem proving. In Alan Robinson and Andrei Voronkov, editors, Handbook of Automated Reasoning, volume 1, chapter 7, pages 371-443. North Holland, 2001.

[15] A. Riazanov and Voronkov A. The design and implementation of Vampire. AI Communications, 15(2-3), 2002.

[16] Stephan Schulz. E-Setheo. http://www4.informatik.tu-muenchen.de/ `schulz/WORK/ e-setheo.html.

[17] Jürgen Stuber. Deriving theory superposition calculi from convergent term rewriting systems. In Proc. 11th Int. Conf. on Rewriting Techniques and Applications (RTA 2000), volume 1833 of $L N C S$, pages 229-245, Norwich, UK, 2000. Springer.

[18] Jürgen Stuber. A model-based completeness proof of Extended Narrowing And Resolution. In Proc. 1st Int. Joint Conf. on Automated Reasoning (IJCAR-2001), LNCS 2083, pages 195-210, Siena, Italy, 2001.

[19] G. S. Tseitin. On the complexity of derivation in propositional calculus, 1970. Reprinted in J. Siekmann and G. Wrightson (eds): Automation of Reasoning, Vol. 2, Springer, 1983, pp. $466-486$.

\section{A An Extended Example}

Using Saturate we prove that the composition "dot" of binary relations is injective in the first argument if the second argument is an injective function. The axiomatization setrel of sets and equations employed includes the axioms numbered 1-15 in the proof below. The 
goal appears as number 16. $\operatorname{rel}(\mathrm{R}, \mathrm{S}, \mathrm{T})$ is true if $\mathrm{R}$ is a binary relation with domain $\mathrm{S}$ and codomain T. Functions from $S$ to $T$ satisfy func $(F, S, T)$. Membership of $X$ in a set $S$ is given by facts of the form contains ( $\mathrm{S}, \mathrm{X})$.

Variables are written as in Prolog. == is an equivalence on formulas used for forming literals, and $=$ is equality on terms. $\Leftrightarrow=>$ and $\Rightarrow$ are, respectively, equivalence and implication at the logical level. Clauses are written as sequents $A_{1}, \ldots, A_{m}->B_{1}, \ldots, B_{n}$. ! is universal, and ? is existential quantification, respectively.

In the proof below, "expansion" denotes steps by one of the CNF expansion rules, and "chaining" indicates superposition inferences. "reduction" stands for simplification steps by contextual rewriting. The proof was done with respect to a manually given precedence on the symbols, and with structural, but eager CNF expansion, preserving equivalences. Skolem functions appear as $\mathbf{s} \$ \mathrm{k}$, and propositions denoting subformulas as they are introduced during CNF expansion steps are written as $\mathrm{q} \$ \mathrm{~m}$. Lines $1,2,4,6,11-15,17,18,53,59$, $60,61,72,84,86$ contain formulas with embedded equivalences. Inferences with these equivalences are computed at lines $17,18,19,53,60,65,84$, and 86. Among these, lines 53 and 84 are steps by superposition of one equivalence into another, whereas the others employ equivalences as rewrite rules in a simplifying manner. As we can see, equivalences are used in the first half of the proof quite extensively, either to expand definitions or for simplification. Boolean reasoning and simplification dominate the second part of the proof. Lines prefixed by "+" denote formulas that have support from the goal clause 16 . As we can see only very few clauses are derived that do not have goal support so that proof search, although saturation-based, is closely goal-oriented. One should also note that only 339 clauses, out of which only 237 clauses were kept, were generated to find this proof of length 111 (where reduction sequences count as single steps) so that every other clause generated was used in the proof. "Search depth" is the depth of superposition inferences (not including simplifications) in the proof tree. "Depth" is the depth of the proof including simplifications. SPASS V2.1 does not find a proof within $2 \mathrm{~h}$ of runtime.

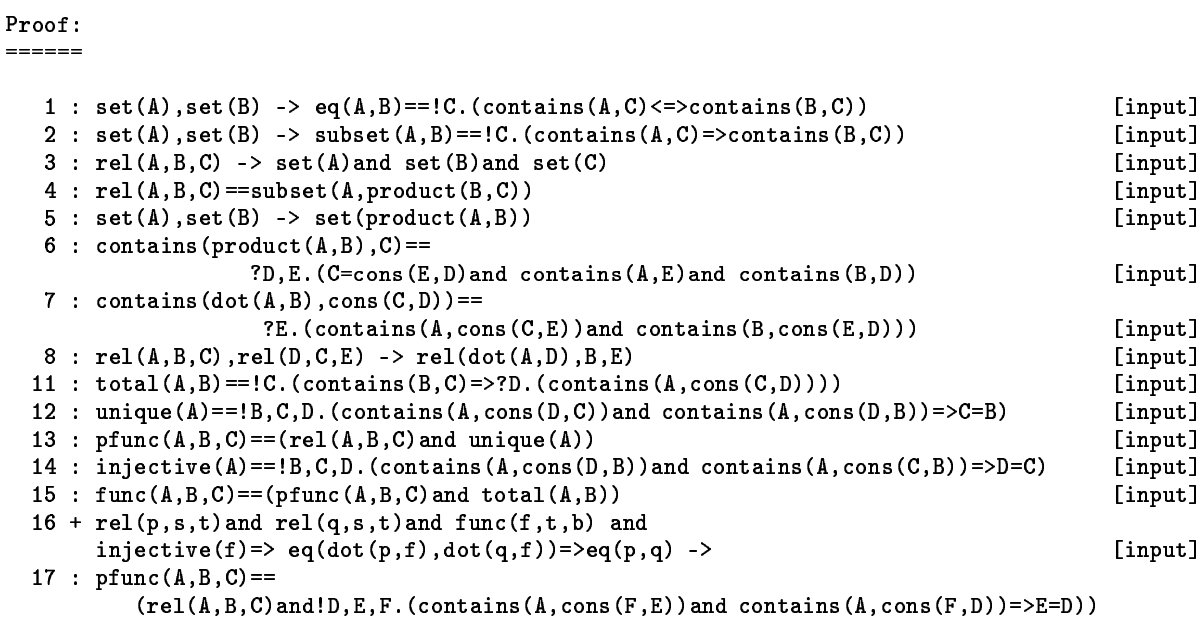


[reduction of 13 by [12]]

18 : $\operatorname{func}(A, B, C)==(\operatorname{rel}(A, B, C)$ and $! D, E, F .(\operatorname{contains}(A, \operatorname{cons}(F, E))$ and contains $(A, \operatorname{cons}(F, D))=>E=D)$ and $! G .(\operatorname{contains}(B, G)=>$ ?.$(\operatorname{contains}(A, \operatorname{cons}(G, H))))$ )

$19+\operatorname{rel}(\mathrm{p}, \mathrm{s}, \mathrm{t})$ and $\operatorname{rel}(\mathrm{q}, \mathrm{s}, \mathrm{t})$ and $(\mathrm{rel}(\mathrm{f}, \mathrm{t}, \mathrm{b})$ and [reduction of 15 by $[11,17]]$

$! A, B, C$. (contains $(f, \operatorname{cons}(C, B))$ and contains $(f, \operatorname{cons}(C, A))=>B=A$ ) and

!D. $(\operatorname{contains}(t, D)=>$ ?E. $(\operatorname{contains}(f, \operatorname{cons}(D, E))))$ ) and

! F, G,H. (contains $(f, \operatorname{cons}(H, F))$ and contains $(f, \operatorname{cons}(G, F))=>H=G) \Rightarrow$

$$
e q(\operatorname{dot}(p, f), \operatorname{dot}(q, f))=>e q(p, q) \rightarrow
$$

[reduction of 16 by $[14,18]]$

[expansion from 3]

$20: \operatorname{rel}(A, B, C) \rightarrow \operatorname{set}(A)$ and $\operatorname{set}(B)$

[expansion from 3]

$22+\operatorname{rel}(p, s, t)$ and $\operatorname{rel}(q, s, t)$ and $(r e l(f, t, b)$ and

$! A, B, C$. (contains $(f, \operatorname{cons}(C, B))$ and contains $(f, \operatorname{cons}(C, A))=>B=A)$ and

!D. $($ contains $(t, D)=>$ ?E. $($ contains $(f, \operatorname{cons}(D, E))))$ ) and

! F, G,H. (contains $(f, \operatorname{cons}(H, F)$ ) and contains $(f, \operatorname{cons}(G, F))=>H=G$ )

$23+e q(\operatorname{dot}(p, f), \operatorname{dot}(q, f))=>e q(p, q)->$

$24: \operatorname{rel}(A, B, C) \rightarrow \operatorname{set}(A)$

$25: \operatorname{rel}(A, B, C) \rightarrow \operatorname{set}(B)$

$26+\operatorname{rel}(p, s, t)$ and $\operatorname{rel}(q, s, t)$ and $(r e l(f, t, b)$ and

! $A, B, C$. (contains $(f, \operatorname{cons}(C, B))$ and contains $(f, \operatorname{cons}(C, A))=B=A$ ) and

!D. ( $\operatorname{contains}(t, D)=>$ ?.$(\operatorname{contains}(f, \operatorname{cons}(D, E)))))$
$27+\operatorname{contains}(f, \operatorname{cons}(A, B)), \operatorname{contains}(f, \operatorname{cons}(C, B)) \rightarrow A=C$

[expansion from 19] [expansion from 19] [expansion from 20] [expansion from 20]

$28+e q(\operatorname{dot}(p, f), \operatorname{dot}(q, f))$

$29+e q(p, q) \rightarrow$

$30+\operatorname{rel}(\mathrm{p}, \mathrm{s}, \mathrm{t})$ and $\operatorname{rel}(\mathrm{q}, \mathrm{s}, \mathrm{t})$

$31+\operatorname{rel}(f, t, b)$ and $! A, B, C$. (contains $(f, \operatorname{cons}(C, B))$ and contains $(f, \operatorname{cons}(C, A))=$

$32+\operatorname{rel}(p, s, t)$ $B=A)$ and! D. (contains $(t, D)=>? E$. (contains $(f, \operatorname{cons}(D, E)))$ )

[expansion from 22] [expansion from 22] [expansion from 23] [expansion from 23] [expansion from 26]

$33+\operatorname{rel}(q, s, t)$

[expansion from 26] [expansion from 30]

$34+\operatorname{rel}(f, t, b)$ and $! A, B, C .(\operatorname{contains}(f, \operatorname{cons}(C, B))$ and contains $(f, \operatorname{cons}(C, A))=>B=A)$

$35+\operatorname{contains}(t, A)->$ ?B. $(\operatorname{contains}(f, \operatorname{cons}(A, B)))$

$36+\operatorname{rel}(f, t, b)$

[expansion from 31] [expansion from 31] [expansion from 34]

$38+\operatorname{contains}(t, A) \rightarrow \operatorname{contains}(f, \operatorname{cons}(A, s \$ 9(f, A)))$

$39+\operatorname{set}(t)$

$42+\operatorname{set}(p)$ [expansion from 35]

$43+\operatorname{set}(q)$

$45+\operatorname{set}(\mathrm{s})$

$49: \operatorname{rel}(A, B, C), \operatorname{rel}(D, C, E) \rightarrow \operatorname{set}(\operatorname{dot}(A, D))$

[negative chaining of 32 from 21]
[negative chaining of 32 from 24]

[negative chaining of 33 from 24]

[negative chaining of 32 from 25] [negative chaining of 8 from 24]

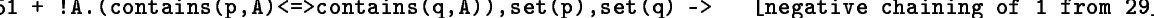
$52+\operatorname{set}(\operatorname{dot}(p, f)), \operatorname{set}(\operatorname{dot}(q, f))->! A .(\operatorname{contains}(\operatorname{dot}(p, f), A)<=>\operatorname{contains}(\operatorname{dot}(q, f), A))$

[chaining of 1 from 28]

$53: \operatorname{set}(A), \operatorname{set}(\operatorname{product}(B, C)) \rightarrow ! D \cdot(\operatorname{contains}(A, D)=>\operatorname{contains}(\operatorname{product}(B, C), D))==\operatorname{rel}(A, B, C)$

[chaining of 2 from 4]

$56+\operatorname{rel}(A, B, t) \rightarrow \operatorname{set}(\operatorname{dot}(A, f))$

[negative chaining of 36 from 49]
[reduction of 51 by $[42,43]]$

$58+!$. (contains $(p, A)<=>$ contains $(q, A)) \rightarrow$

$59+\operatorname{set}(\operatorname{dot}(p, f)), \operatorname{set}(\operatorname{dot}(q, f)) \rightarrow \operatorname{contains}(\operatorname{dot}(p, f), A)==\operatorname{contains}(\operatorname{dot}(q, f), A)$

$60: \operatorname{set}(A), \operatorname{set}(\operatorname{product}(B, C)) \rightarrow ! D .(\operatorname{contains}(A, D)=>? E, F .(D=\operatorname{cons}(F, E)$ and $\operatorname{contains}(B, F)$ and contains $(C, E)))==\operatorname{rel}(A, B, C) \quad$ [reduction of 53 by [6]]

$61+\operatorname{contains}(\mathrm{p}, \mathrm{s} \$ 16)==$ contains $(\mathrm{q}, \mathrm{s} \$ 16)->$

[expansion from 58]

64 : $\operatorname{contains}(A, B), \operatorname{rel}(A, C, D), \operatorname{set}(A), \operatorname{set}(\operatorname{product}(C, D))$

$\rightarrow$ ?E, F. (B=cons $(F, E)$ and contains $(C, F)$ and contains $(D, E))$ [expansion from 60]

$65+$ contains ( $q, s \$ 16)$ and contains $(p, s \$ 16), \sim \operatorname{contains~(q,s} \$ 16)$ and contains $(p, s \$ 16)$

$68+\operatorname{set}(\operatorname{dot}(p, f))$

$69+\operatorname{set}(\operatorname{dot}(q, f))$

71 : $\operatorname{set}(\operatorname{product}(A, B)), \operatorname{rel}(C, A, B), \operatorname{contains}(C, D)->$

?E, F. ( $D=\operatorname{cons}(F, E)$ and contains $(A, F)$ and contains $(B, E)) \quad$ [reduction of 64 by [24]]

$72+\operatorname{contains}(\operatorname{dot}(q, f), A)==\operatorname{contains}(\operatorname{dot}(p, f), A)$

$74: \operatorname{rel}(A, B, C), \operatorname{set}(\operatorname{product}(B, C)), \operatorname{contains}(A, D) \rightarrow$

?E. $(D=\operatorname{cons}(E, s \$ 4(B, C, D))$ and contains $(B, E)$ and contains $(C, s \$ 4(B, C, D)))$ 


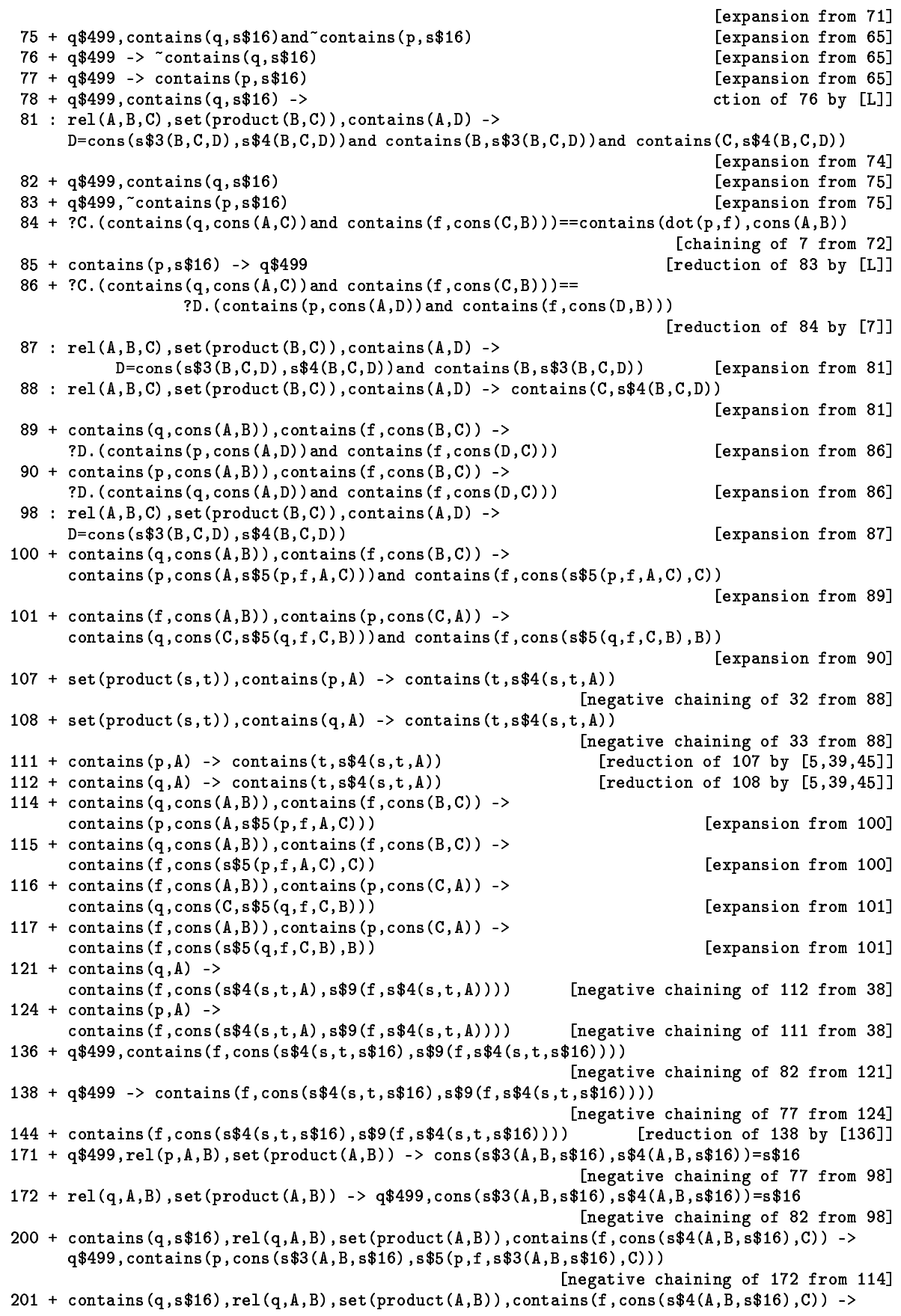


$\mathrm{q} \$ 499$, contains ( $\mathrm{f}, \mathrm{cons}(\mathrm{s} \$ 5(\mathrm{p}, \mathrm{f}, \mathrm{s} \$ 3(\mathrm{~A}, \mathrm{~B}, \mathrm{~s} \$ 16), \mathrm{C}), \mathrm{C})$ ) [negative chaining of 172 from 115 ]

$205+$ contains $(f, \operatorname{cons}(s \$ 4(A, B, s \$ 16), C)), \operatorname{set}(\operatorname{product}(A, B)), \operatorname{rel}(q, A, B) \rightarrow$ contains ( $p$, cons ( $\$ 3(A, B, s \$ 16), s \$ 5(p, f, s \$ 3(A, B, s \$ 16), C))$ ) , $\$ \$ 499$

[reduction of 200 by [82]]

$206+$ contains $(f, \operatorname{cons}(s \$ 4(A, B, s \$ 16), C)), \operatorname{set}(\operatorname{product}(A, B)), \operatorname{rel}(q, A, B)->$ contains $(f, \operatorname{cons}(s \$ 5(p, f, s \$ 3(A, B, s \$ 16), C), C)), q \$ 499$ [reduction of 201 by [82]]

$215+\operatorname{set}(\operatorname{product}(A, B)), \operatorname{rel}(q, A, B), \operatorname{contains}(f, \operatorname{cons}(s \$ 4(A, B, s \$ 16), C))$, contains $(f, \operatorname{cons}(D, C)) \rightarrow q \$ 499, D=s \$ 5(p, f, s \$ 3(A, B, s \$ 16), C)$

[negative chaining of 206 from 27]

$228+\operatorname{contains}(f, \operatorname{cons}(A, s \$ 9(f, s \$ 4(s, t, s \$ 16)))), \operatorname{set}(\operatorname{product}(s, t)), \operatorname{rel}(q, s, t) \rightarrow$ $\mathrm{q} \$ 499, \mathrm{~A}=\mathrm{s} \$ 5(\mathrm{p}, \mathrm{f}, \mathrm{s} \$ 3(\mathrm{~s}, \mathrm{t}, \mathrm{s} \$ 16), \mathrm{s} \$ 9(\mathrm{f}, \mathrm{s} \$ 4(\mathrm{~s}, \mathrm{t}, \mathrm{s} \$ 16))$ ) [negative chaining of 144 from 215]

233 + contains $(f, \operatorname{cons}(A, s \$ 9(f, s \$ 4(s, t, s \$ 16)))) \rightarrow$

$A=\mathbf{s} \$ 5(p, f, s \$ 3(s, t, s \$ 16), \mathbf{s} \$ 9(f, s \$ 4(s, t, s \$ 16))), q \$ 499$ [reduction of 228 by $[5,39,45,33]$ $239+s \$ 4(s, t, s \$ 16)=s \$ 5(p, f, s \$ 3(s, t, s \$ 16), s \$ 9(f, s \$ 4(s, t, s \$ 16))), q \$ 499$

[negative chaining of 144 from 233]

$259+\operatorname{set}(\operatorname{product}(\mathrm{s}, \mathrm{t})), \operatorname{rel}(\mathrm{q}, \mathrm{s}, \mathrm{t})$, contains $(\mathrm{f}, \operatorname{cons}(\mathrm{s} \$ 4(\mathrm{~s}, \mathrm{t}, \mathrm{s} \$ 16), \mathrm{s} \$ 9(\mathrm{f}, \mathrm{s} \$ 4(\mathrm{~s}, \mathrm{t}, \mathrm{s} \$ 16)))$ ) $\rightarrow$ contains ( $p$, cons $(s \$ 3(s, t, s \$ 16), s \$ 4(s, t, s \$ 16))), q \$ 499, q \$ 499$ [chaining of 239 from 205] $260+\operatorname{set}(\operatorname{product}(s, t)), \operatorname{rel}(q, s, t), \operatorname{contains}(f, \operatorname{cons}(s \$ 4(s, t, s \$ 16), s \$ 9(f, s \$ 4(s, t, s \$ 16)))) \rightarrow$ contains ( $p$, cons $(s \$ 3(s, t, s \$ 16), s \$ 4(s, t, s \$ 16))$ ), $q \$ 499$ [condensement of 259]

$261+\mathrm{q} \$ 499 \quad$ [reduction of 260 by $[5,39,45,33,144,172,5,39,45,33,85, \mathrm{~L}]$

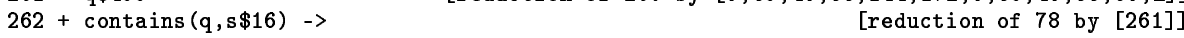

$263+$ contains $(\mathrm{p}, \mathrm{s} \$ 16) \quad$ [reduction of 77 by [261]]

$264+\operatorname{rel}(p, A, B), \operatorname{set}(\operatorname{product}(A, B)) \rightarrow$ $\operatorname{cons}(\mathbf{s} \$ 3(A, B, \mathbf{s} \$ 16), \mathbf{s} \$ 4(A, B, \mathbf{s} \$ 16))=\mathbf{s} \$ 16 \quad$ [reduction of 171 by [261]]

$273+\operatorname{contains}(p, s \$ 16), \operatorname{rel}(p, A, B), \operatorname{set}(\operatorname{product}(A, B)), \operatorname{contains}(f, \operatorname{cons}(s \$ 4(A, B, s \$ 16), C)) \rightarrow$ contains $(f, \operatorname{cons}(s \$ 5(q, f, s \$ 3(A, B, s \$ 16), C), C)$ [negative chaining of 264 from 117]

$277+\operatorname{contains}(f, \operatorname{cons}(s \$ 4(A, B, s \$ 16), C)), \operatorname{set}(\operatorname{product}(A, B)), \operatorname{rel}(p, A, B)->$ contains $(f$, cons $(s \$ 5(q, f, s \$ 3(A, B, s \$ 16), C), C))$ [reduction of 273 by [263]] $280+\operatorname{set}(\operatorname{product}(A, B)), \operatorname{rel}(p, A, B), \operatorname{contains}(f, \operatorname{cons}(s \$ 4(A, B, s \$ 16), C)), \operatorname{contains}(f, \operatorname{cons}(D, C)) \rightarrow$ $\mathrm{D}=\mathbf{s} \$ 5(\mathrm{q}, \mathrm{f}, \mathbf{s} \$ 3(\mathrm{~A}, \mathrm{~B}, \mathbf{s} \$ 16), \mathrm{C}) \quad$ [negative chaining of 277 from 27]

$293+\operatorname{contains}(f, \operatorname{cons}(A, s \$ 9(f, s \$ 4(s, t, s \$ 16)))), \operatorname{set}(\operatorname{product}(s, t))$, $\operatorname{rel}(p, s, t) \rightarrow A=s \$ 5(q, f, s \$ 3(s, t, s \$ 16), s \$ 9(f, s \$ 4(s, t, s \$ 16)))$

$298+\operatorname{contains}(f, \operatorname{cons}(A, s \$ 9(f, s \$ 4(s, t, s \$ 16)))) \rightarrow$

[negative chaining of 144 from 280] $A=s \$ 5(q, f, s \$ 3(s, t, s \$ 16), s \$ 9(f, s \$ 4(s, t, s \$ 16)))$ $303+\mathrm{s} \$ 4(\mathrm{~s}, \mathrm{t}, \mathrm{s} \$ 16)=\mathrm{s} \$ 5(\mathrm{q}, \mathrm{f}, \mathrm{s} \$ 3(\mathrm{~s}, \mathrm{t}, \mathrm{s} \$ 16), \mathrm{s} \$ 9(\mathrm{f}, \mathrm{s} \$ 4(\mathrm{~s}, \mathrm{t}, \mathrm{s} \$ 16)))$ [reduction of 293 by $[5,39,45,32]]$

[negative chaining of 144 from 298]

$315+\operatorname{contains}(f, \operatorname{cons}(A, s \$ 9(f, s \$ 4(s, t, s \$ 16))))$, contains $(p, \operatorname{cons}(s \$ 3(s, t, s \$ 16), A)) \rightarrow$ contains ( $q$, cons ( $\$ 3(s, t, s \$ 16), s \$ 4(s, t, s \$ 16))$ ) [chaining of 303 from 116] $318+\operatorname{contains}(f, \operatorname{cons}(A, s \$ 9(f, s \$ 4(s, t, s \$ 16))))$, contains $(p, \operatorname{cons}(s \$ 3(s, t, s \$ 16), A)) \rightarrow$

$322+\operatorname{contains}(p, s \$ 16), \operatorname{rel}(p, s, t), \operatorname{set}(\operatorname{product}(s, t))$ reduction of 315 by $[264,5,39,45,32,262, \mathrm{~L}]]$ contains ( $f$, cons $(s \$ 4(s, t, s \$ 16), s \$ 9(f, s \$ 4(s, t, s \$ 16)))$ ) $\rightarrow$

$339+$ false

[negative chaining of 264 from 318]

Length $=111$, Depth $=37$, Search Depth $=8$

Total time: 13010 milliseconds

Number of forward inferences: 189

Number of tableau inferences: 71

Number of generated clauses: 339

Number of kept clauses: 237 


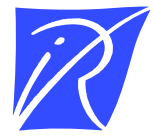

Unité de recherche INRIA Lorraine

LORIA, Technopôle de Nancy-Brabois - Campus scientifique 615, rue du Jardin Botanique - BP 101 - 54602 Villers-lès-Nancy Cedex (France)

Unité de recherche INRIA Futurs : Parc Club Orsay Université - ZAC des Vignes

4, rue Jacques Monod - 91893 ORSAY Cedex (France)

Unité de recherche INRIA Rennes : IRISA, Campus universitaire de Beaulieu - 35042 Rennes Cedex (France)

Unité de recherche INRIA Rhône-Alpes : 655, avenue de l'Europe - 38334 Montbonnot Saint-Ismier (France) Unité de recherche INRIA Rocquencourt : Domaine de Voluceau - Rocquencourt - BP 105 - 78153 Le Chesnay Cedex (France)

Unité de recherche INRIA Sophia Antipolis : 2004, route des Lucioles - BP 93 - 06902 Sophia Antipolis Cedex (France)

INRIA - Domaine de Voluceau - Rocquencourt, BP 105 - 78153 Le Chesnay Cedex (France)

http://www.inria.fr

ISSN 0249-6399 